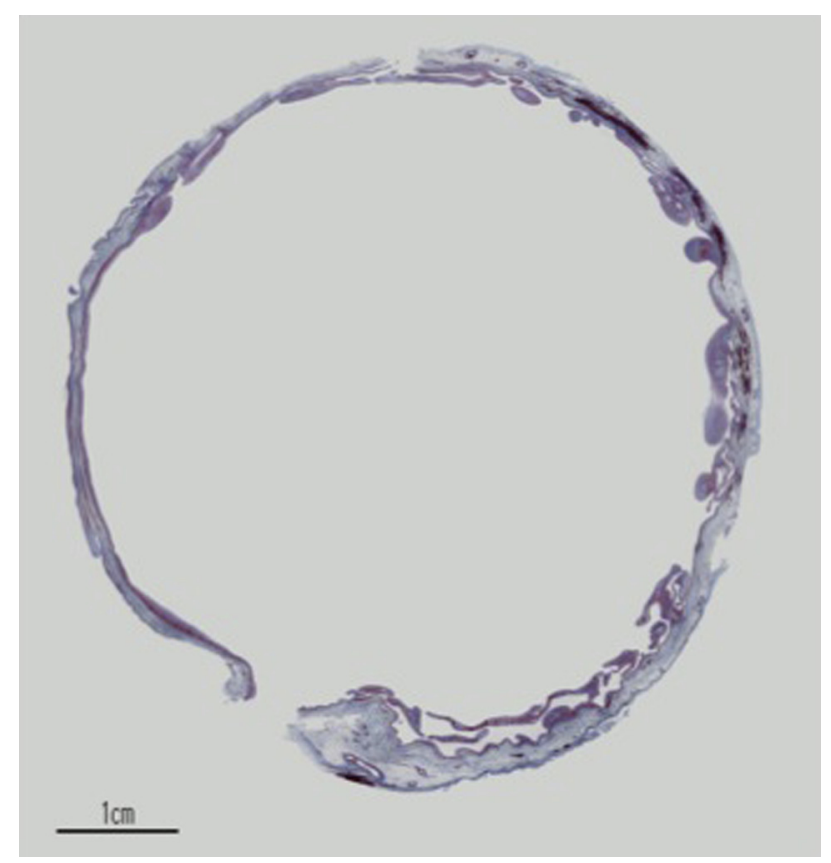

FIGURE 2. Histologic findings of the explanted heart showing an extremely thin left ventricular wall with marked fibrosis (elastica van Gieson's stain).

function. Furthermore, an exercise stress test showed increased left VAD flow even at a fixed pump speed. Frazier et $\mathrm{al}^{2}$ used a calf model in which both native ventricles were excised and replaced with 2 continuous-flow VADs. An exercise test demonstrated increased flow in both VADs. ${ }^{2}$ Our results have confirmed their experimental findings, suggesting that even without native ventricular contraction, biventricular VADs can adjust their flow according to the flow demand. We consider that these hemodynamic properties imply the possibility of clinical use of continuous-flow artificial hearts currently being tested in animal models.

When implanting 2 rotary pumps for biventricular support, concern exists about a flow imbalance between the pulmonary and systemic circulation. After the implant operation, the systemic and pulmonary vascular resistances become dynamic and are further influenced by use of inotropes, discontinuation of mechanical ventilation, and other factors. These altered hemodynamic states could exacerbate the flow imbalance during the perioperative period, especially in patients with a noncontractile heart. In the present case, the first extubation failed owing to severe pulmonary congestion caused by right VAD overflow. ${ }^{3}$ Interestingly, after resolution of these perioperative hemodynamic fluxes, the management became easier, with no additional pulmonary congestion noted. A natural flow balance appears to be achieved by the autoregulatory properties of continuous-flow VADs. ${ }^{4}$

The currently available pulsatile total artificial hearts have numerous restrictions. We believe that the present successful case will promote the clinical use of rotary blood pumps in patients with biventricular failure for bridging and, even, destination therapy.

\section{References}

1. Krabatsch T, Potapov E, Stepanenko A, Schweiger M, Kukucka M, Huebler M, et al. Biventricular circulatory support with two miniaturized implantable assist devices. Circulation. 2011;124:S179-86.

2. Frazier OH, Cohn WE, Tuzon E, Winkler JA, Gregoric ID. Continuous flow total artificial heart supports long-term survival of a calf. Tex heart Inst J. 2009;36:568-74.

3. Saito S, Sakaguchi T, Miyagawa S, Yoshikawa Y, Yamauchi T, Ueno T, et al. Biventricular support using implantable continuous-flow ventricular assist devices. J Heart Lung Transplant. 2011;30:475-8.

4. Westaby S, Frazier OH. Long-term biventricular support with rotary blood pumps: prospects and pitfalls. Eur J Cardiothorac Surg. 2012;42:203-8.

\title{
Acquired von Willebrand disease in a child with a ventricular assist device
}

\author{
Karine Nubret, MD, ${ }^{\mathrm{a}}$ Philippe Mauriat, MD, ${ }^{\mathrm{a}}$ François Roubertie, MD, ${ }^{\mathrm{b}}$ Chloé James, MD, $, \mathrm{d}, \mathrm{e}$ \\ Nadir Tafer, MD, ${ }^{\mathrm{a}}$ and Alexandre Ouattara, $\mathrm{MD}, \mathrm{PhD},{ }^{\mathrm{a}, \mathrm{d}, \mathrm{e}}$ Bordeaux and Pessac, France
}

\footnotetext{
From Service d'Anesthésie-Réanimation II, ${ }^{\mathrm{a}}$ Service de Chirurgie Cardiovasculaire, ${ }^{\mathrm{b}}$ and Laboratoire d'Hématologie-Hémostase, ${ }^{\mathrm{c}} \mathrm{CHU}$ de Bordeaux, Bordeaux, France; and Adaptation Cardiovasculaire à l'Ischémie, ${ }^{\mathrm{d}}$ University of Bordeaux, and Adaptation Cardiovasculaire à l'Ischémie, ${ }^{\mathrm{e}}$ INSERM, Pessac, France.

Disclosures: Authors have nothing to disclose with regard to commercial support.

Received for publication Feb 3, 2013; revisions received April 21, 2013; accepted for publication June 18, 2013; available ahead of print July 26, 2013.

Address for reprints: Alexandre Ouattara, MD, PhD, Service d'AnesthésieRéanimation 2, Hôpital Haut-Lévèque-CHU de Bordeaux, Avenue de Magellan, F-33604 Pessac, France (E-mail: alexandre.ouattara@chu-bordeaux.fr).

J Thorac Cardiovasc Surg 2013;146:e30-2

0022-5223/\$36.00

Copyright (C) 2013 by The American Association for Thoracic Surgery

http://dx.doi.org/10.1016/j.jtcvs.2013.06.023
}

The ventricular assist device (VAD), as a bridge to heart transplantation, is being increasingly used in patients of all ages who are experiencing refractory heart failure. In these patients, massive bleeding remains a leading and challenging complication. Among the possible causes, the occurrence of acquired von Willebrand disease (avWD) has been reported in adult patients. ${ }^{1}$ Indeed, the loss of high-molecular-weight (HMW) von Willebrand factor (vWF) multimers because of shear stress, similar to that seen in aortic stenoses, ${ }^{1}$ is frequently observed in patients with axial flow in a left VAD. ${ }^{2,3}$ 


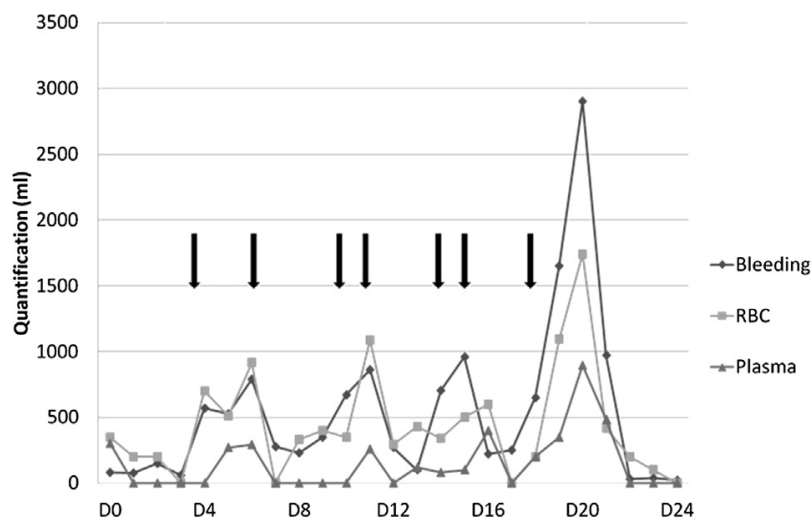

FIGURE 1. Bleeding and allogenic transfusions. Quantification of bleeding, and red blood cell $(R B C)$ counts and plasma requirements, after implantation of a biventricular assist device. Arrows, Surgical re-explorations; $D$, postoperative day.

To treat bleeding associated with avWD, various therapies, including reduced anticoagulation, the control of local bleeding, and plasma or platelet transfusions, are used. However, none of these therapies specifically address the abnormalities seen in avWD, and the effectiveness of these therapies remains uncertain. Herein, we describe the first case of avWD in a pediatric patient after implantation of a pulsatile biventricular assist device (BerlinHeart; Berlin Heart GmBH, The Woodlands, Tex), which caused severe perioperative bleeding but could be treated with von Willebrand factor concentrate. The child was successfully bridged until heart transplantation.

\section{CASE REPORT}

A 3-year-old, 13-kg boy, with a history of dilated cardiomyopathy, had a biventricular assist device implanted as a bridge until heart transplantation. The antithrombotic regimen consisted of only unfractionated heparin. Four days after the device was implanted, a bleed of 5 $\mathrm{mL} \cdot \mathrm{kg}^{-1} \cdot \mathrm{h}^{-1}$ occurred, which required surgical reexploration. A 700-mL red blood cell (RBC) transfusion was also necessary. By day 5 , a bleed of $2 \mathrm{~mL} \cdot \mathrm{kg}^{-1} \cdot \mathrm{h}^{-1}$ persisted and required transfusion of $500 \mathrm{~mL}$ of $\mathrm{RBCs}$, $270 \mathrm{~mL}$ of plasma, $110 \mathrm{~mL}$ of platelets, and further surgical re-exploration. Despite these therapeutic measures, bleeding recurred and required several surgical reexplorations at 3-day intervals and allogenic transfusion of $9490 \mathrm{~mL}$ of RBCs and $3620 \mathrm{~mL}$ of plasma (Figure 1).

Prothrombin time and fibrinogen levels were closely monitored. ROTEM monitoring (Tem International $\mathrm{GmbH}$, München, Germany) showed an EXTEM clotting time value of 66 seconds (normal range, 46-97 seconds), an EXTEM clotting formation time value of 196 seconds (normal range, 41-109 seconds), an $\alpha$ angle of $62^{\circ}$ (normal range, $69^{\circ}-82^{\circ}$ ), and maximal clot firmness of $43 \mathrm{~mm}$ (normal range, 52-70 $\mathrm{mm})$. By day 20, the patient developed increased

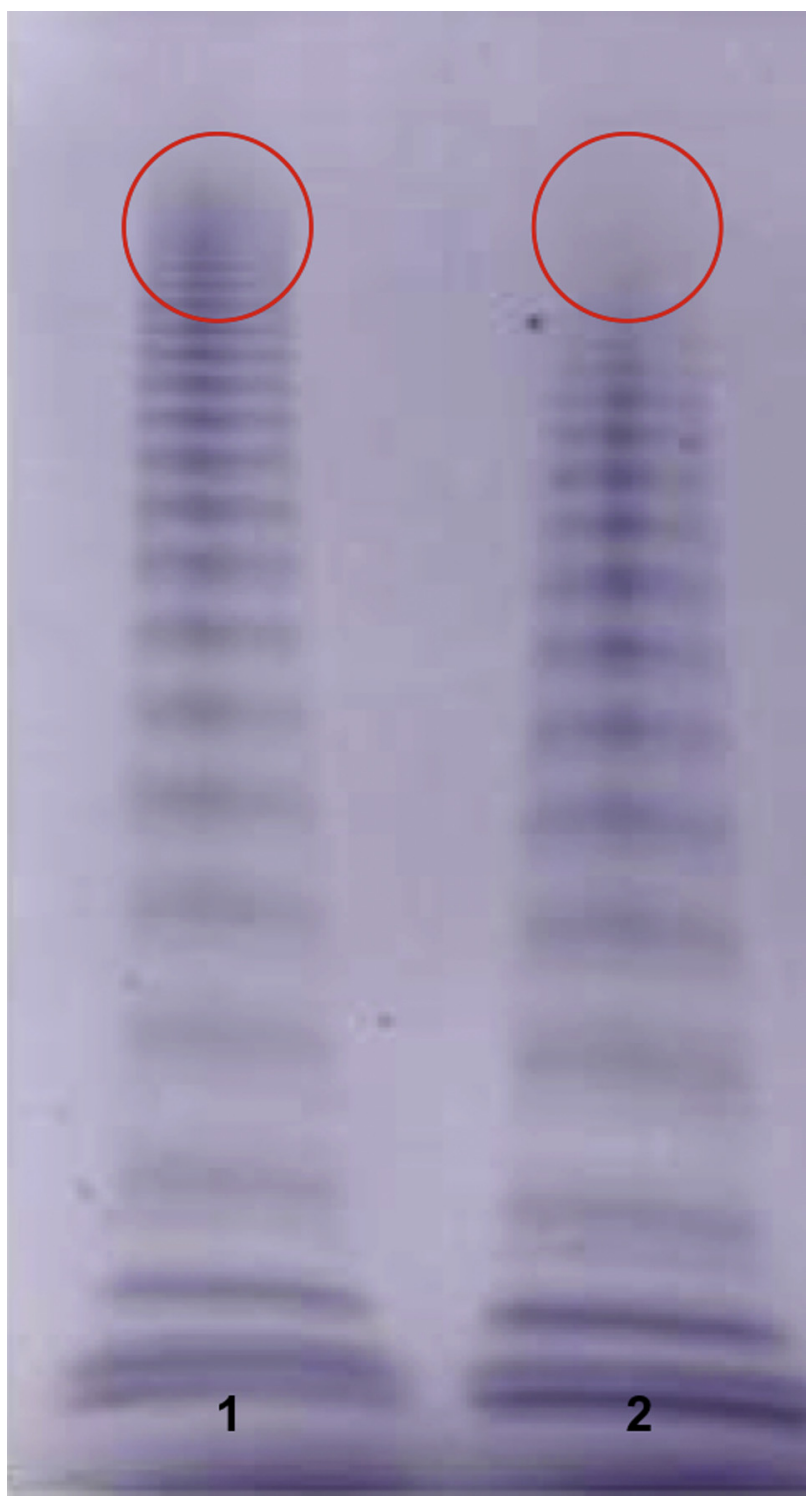

FIGURE 2. Normal plasma (lane 1) and plasma from our patient receiving a biventricular assist device (lane 2). The dye front is at the bottom of the discontinuous gel, and the large multimers are shown in the upper part of the gel. Lane 1 indicates normal plasma; lane 2, plasma from our patient who had lost high-molecular-weight multimers, as indicated by the missing bands in the upper part of the gel (red circle).

hemorrhagic syndrome $\left(9 \mathrm{~mL} \cdot \mathrm{kg}^{-1} \cdot \mathrm{h}^{-1}\right)$ with an increased requirement for transfusion of $1740 \mathrm{~mL}$ of RBCs, $895 \mathrm{~mL}$ of plasma, and $438 \mathrm{~mL}$ of platelets. Because bleeding was severe, an infusion of tranexamic acid $\left(3 \mathrm{~mL} \cdot \mathrm{kg}^{-1} \cdot \mathrm{h}^{-1}\right)$ was given. The patient also received 3 doses $(30 \mu \mathrm{g} / \mathrm{kg})$ of recombinant activated factor VII, although these were unsuccessful (Novoseven; NovoNordisk, Princeton, NJ). We also stopped heparin for 3 hours while heparin activity was within normal limits (anti-Xa activity, 0.2-0.3 IU/mL).

Because avWD was suspected, human vWF concentrates of $80 \mathrm{IU} / \mathrm{kg}$ every 8 hours, associated with human factor 
VIII (FVIII) concentrates of $40 \mathrm{IU} / \mathrm{kg}$ (Wilstart; LFB Biomédicaments, Les Ulis, France) every 8 hours, were started. Before giving these new therapies, blood was collected for an assay. The following day, the daily requirement for RBC transfusion had decreased to $400 \mathrm{~mL}$ (Figure 1). The results of the von Willebrand panel showed that the vWF antigen (vWF:Ag) was normal ( $77 \%$ by enzyme-linked immunosorbent assay; normal range, $50 \%-160 \%$ ), as was factor VIII coagulant ( $62 \%$ vs normal range, 60\%-150\%). Collagen-binding activity (vWF:CB) was $58 \%$ by enzyme-linked immunosorbent assay (normal range, $50 \%-150 \%$ ), as was vWF ristocetin cofactor $(64 \%$ vs normal range, $60 \%-130 \%)$. The ratio of vWF:CB/vWF:Ag was 0.75 , which was also within the normal range $(\geq 0.70)$. Analysis of vWF multimers revealed an abnormal pattern, mainly characterized by a marked decrease in HMW forms, which was compatible with avWD (Figure 2). Because FVIII coagulant was normal, only therapy with human vWF concentrate was continued (Wilfactin; LFB Biomédicaments) and at the same dose. At 72 hours after beginning this therapy, the child did not require any further transfusion support until heart transplantation could be performed (on day 29). There were no thromboembolic complications. The postoperative course was uneventful, and the patient left the intensive care unit a week after heart transplantation.

\section{DISCUSSION}

To our knowledge, this is the first case of avWD to be described in a child, although avWD has been previously reported in adults after a VAD implantation. Heilmann and colleagues ${ }^{4}$ analyzed patients with a pneumatic VAD system or an axial flow pump for the presence of avWD and for bleeding complications. They observed bleeding episodes more frequently in VAD than in axial flow pump, in the early and the late course. However, they did not report any significant difference in the severity of the avWD. An avWD results from shear stress in the pump itself and in the tubes that connect the pump to the heart and great vessels. This shear stress causes unfolding of the HMW multimers of vWF with exposure of the D2 domains of the VWF monomers and subsequent cleavage by the protease ADAMTS- $13 .{ }^{5}$ Bleeding phenomenon may be attributed to the loss of large vWF multimers, leading to abnormalities in platelet adhesion at the sites of vascular injury. The bleeding diatheses seen in these patients is out of proportion to that expected with postsurgical anticoagulation, and has been attributed to the additional hemostatic defect contributed by degradation of vWF.

In some patients, a loss or decrease of HMW multimers is the only laboratory abnormality. Various therapies, such as desmopressin acetate, tranexamic acid, and control of local bleeding, have been traditionally used to treat major bleeding events during avWD. However, these therapies do not specifically address the abnormalities observed during avWD. Furthermore, the efficacy of these agents in patients with avWD secondary to VAD is unknown. In our case, many therapies, including recombinant FVIIa, were used in association with large-volume blood and plasma transfusions, but were ineffective. For this reason, we tried a more specific strategy that focused on the missing vWD factor.

Wilstart and Wilfactin (LFB Biomédicaments) are established treatment options for patients with vWD but are not usually prescribed for avWD. Before initiating this treatment, a von Willebrand panel was conducted to obtain values for vWF activity, vWF:Ag, and FVIII when bleeding was the most abundant. An absence of high-molecularweight multimers was the only abnormal factor identified. Because hemorrhagic syndrome was not controlled, we wanted to provide the patient with the missing vWF. Once treatment with Wilfactin was started, we observed a significant decrease in the need for blood transfusions until heart transplantation was conducted. The average daily cost of this therapy was estimated at approximately $€ 3210$ (US\$4220).

In conclusion, because avWD can also occur in children with VAD, our approach should be considered every time there is persistent bleeding. To our knowledge, we have reported the first case of severe bleeding related to avWD in a child supported with a bi-VAD and in whom bleeding was stopped with the use of Wilfactin. Its use in VAD-associated avWD has not been reported previously, and this treatment should be explored further in children with avWD.

We thank Erwan Floch, PharmD (Newmed Publishing Services), for reviewing the manuscript.

\section{References}

1. Vincentelli A, Susen S, Le Tourneau T, Six I, Fabre O, Juthier F, et al. Acquired von Willebrand syndrome in aortic stenosis. N Engl J Med. 2003;349:343-9.

2. Malehsa D, Meyer A, Bara C, Strüber M. Acquired von Willebrand syndrome after exchange of the Heart Mate XVE to the Heart Mate II ventricular assist device. Eur J Cardiothorac Surg. 2009;35:1091-3.

3. Geisen U, Heilmann C, Beyersdorf F, Benk C, Berchtold-Herz M, Schlensak C, et al. Non-surgical bleeding in patients with ventricular assist devices could be explained by acquired von Willebrand disease. Eur J Cardiothorac Surg. 2008; 33:679-84.

4. Heilmann C, Geisen U, Beyersdorf F, Nakamura L, Trummer G, BerchtoldHerz M, et al. Acquired von Willebrand syndrome is an early-onset problem in ventricular assist device patients. Eur J Cardiothorac Surg. 2011;40:1328-33.

5. Balfauf C, Schneppenheim R, Stacklies W, Obser T, Pieconka A, Schneppenheim S, et al. Shear-induced unfolding activates von Willebrand factor A2 domain for proteolysis. J Thromb Haemost. 2009;7:2096-105. 